$2^{\circ}$ Dans le cas des laits colostraux de femme, l'échantillon-type de comparaison est d'un bleu moins intense mais l'aspect macroscopique d'un tel lait suffit à le diagnostiquer et ne permet pas la confusion avec un lait normal mouillé.

$3^{0}$ La substitution de solutions colorantes-types, plus stables, aux échantillons témoins, sujets à des variations de colorations avec le temps, est à l'étude en ce moment; elle fera l'objet d'une prochaine communication.

$4^{0}$ Enfin quelques mots sur la précision de la méthode :

Le procédé permet de déceler une fraude de $5 \%$ sur du lait de femme soit par adjonction de lait de vache soit de lait de chèvre. Cette précision semble suffisante car il ne serait d'aucun profit pour une personne de frauder à des taux inférieurs.

\title{
DOSAGE DES ACIDES DE FERMENTATION ET DE CERTAINES BASES DU LAIT
}

\author{
par

\section{Etienne GOIFFON} \\ Ingénieur I.A.N.
}

Un des principaux problèmes qui se présentent à l'industrie du lait est la fermentation lactique aux dépens du lactose. Le lait, grâce à l'abondance de ce sucre et à la présence de polypeptides abondants, est un bouillon de culture idéal pour les bacilles lactiques, dont l'action est très rapide. C'est contre cela que luttent la stérilisation par la chaleur, le ralentissement de la vie microbienne par le froid, l'accélération du transport, pour lutter de vitesse avec les germes.

Il est indispensable, à toutes les étaṕes de la conservation et du transport du lait, de connaître exactement où en est la progression de cette fermentation, qui tend si rapidement à rendre le lait impropre à la consommation.

On dispose pour cela de deux moyens excellents et pratiques, c'est la mesure plus ou moins approchée du $p \mathbf{H}$, et le dosage de l'acidité conférée au lait par la formation de l'acide lactique.

Mais il est un moyen de corriger cette acidité que ne manquent pas d'employer certains producteurs, industriels ou commerçants, c'est de neutraliser l'acidité formée par l'adjonction au lait d'un alcalin. Le lactate de soude ainsi formé échappe aux mesures d'acidité, et le lait est apparemment normal, pendant un certain laps de temps, quoiqu'il soit réellement le siège d'une pullulation microbienne intense. 
Il serait done désirable d'avoir un procédé qui permît de mesurer l'acide lactique, qu'il soit libre ou salifié et de pouvoir apprécier la présence d'une quantité anormale de bases ajoutées frauduleusement.

C'est ce que nous nous sommes proposé de faire d'une façon plus compliquée que pour le dosage titrimétrique de l'acidité, mais encore très accessible aux industriels dont le laboratoire est très simplifié.

Il s'agit d'abord du dosage des acides faibles du lait, en dehors de l'acide phosphorique. En réalité, c'est l'acide lactique qui sera essentiellement mesuré ; puis nous apprécierons la quantité de bases liées aux acides faibles, en dehors de celles qui font partie des sels tels que le chlorure de sodium.

$$
*^{* *}
$$

Van Slyke et Palmer (1) ont étudié un procédé de dosage des acides organiques urinaires très simple, qui a été étudié depuis par $R$, Golffon et Nepveux (2). C'est une adaptation de cette méthode au lait que nous avons mise au point. Le principe en est le suivant :

Si l'on défèque le liquide biologique que l'on veut employer de façon que les acides organiques restent en solution, on amène une partie aliquote du filtrat à $p H$ 8, au virage de la phénolphtaléine. On y verse en affusion progressive de l'acide chlorhydrique titré jusqu'à ce que le $p H$ de la solution soit à 2,7 (teinte orangée à l'Orange IV). Les affusions d'HCL, acide à forte constante de dissociation ne réussissent qu'à former un chlorure alcalin en mettant en liberté une quantité correspondante d'acides organiques; et cette libération d'acides organiques s'opérera jusqu'à décomposition totale des sels organo-calciques. Or, ces acides organiques sont des acides faibles, c'est-à-dire qu'une partie seulement des ions hydrogènes qu'ils renferment sont à l'état libre, l'autre partie reste combinée, et par conséquent inactive au point de vue de la réaction. L'acidité ionique de ces solutions ne correspond dono pas exactement à leur masse totale, elle n'en est qu'une fraction, déterminée d'ailleurs par la loi des masses. Cette fraction est toujours la même pour chaque acide, e'est une constante. Aux concentrations habituelles des acides organiques dans les liquides biologiques, même quand ils sont libres, la masse des ions $H$ n'atteint jamais la coneentration en hydrions exprimée par $p$ H 2,7.

L'acide chlorhydrique, acide fort, est presque totalement dissocié, une faible quantité $\left(1 \mathrm{~cm}^{2}\right)$ de solution N/10 suffit à amener

(1) Journal of Biol. Chem., t. XII, p. 567.

(2) C. B. Soc. Biol, 1922, 86, 1132. 
$60 \mathrm{~cm}^{3}$ d'eau distillée à cette concentration en $\mathrm{H}$. Aussitốt done que la base de tous les sels d'acides organiques est complètement transformée en chlorure, un léger excès d'HCL amènera très rapidement la solution à $p$ H 2,7 (ce qu'on reconnaît au virage de l'Orangé IV). La quantité d'HCL décime employée mesure done la quantité d'acides organiques libérés, moins $1 \mathrm{~cm}^{2} 2$ nécessaire pour amener $60 \mathrm{~cm}^{3}$ jusqu'au point de virage de l'indicateur.

Courbe de titration, par HCL $\mathrm{N} / 10$, de $10 \mathrm{~cm}^{3}$ d'une solution d'acétate de chaux N/10 (pointillé).

Courbe fournie-par $1 \mathrm{~cm}^{3}$ 2 d'HCL N/10 dans $60 \mathrm{~cm}^{3}$ d'eau (trait plein).

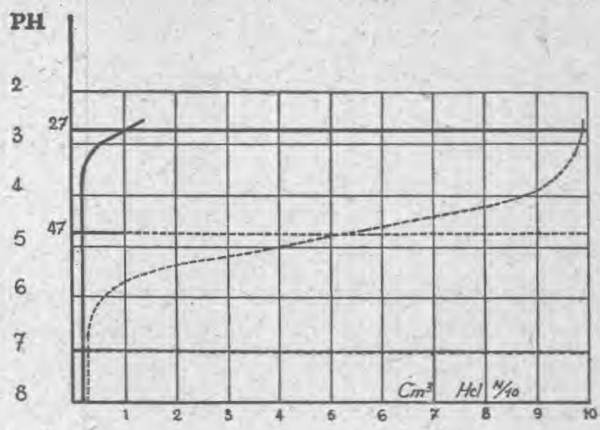

VAN Slyke et Palmer ont montré que $99 \%$ des acides acétique, butyrique, etc., sont théoriquement retrouvés. L'acide lactique n'est dosé que pour $93 \%$, sa constante de dissociation étant relativement élevée. Les bases faibles du lait peuvent intervenir dans la titration : l'ammoniaque, dans la proportion de 5 à $6 \%$; la créatinine et la créatine sont complètement dosées avec les acides.

Les acides aminés sont également dosés dans une plus faible proportion. On peut estimer qu'ils sont neutralisés par un volume d'HCL décime égal au nombre de centigrammes d'azote aminé multiplié par le coefficient 3,2 .

$$
*^{*} *
$$

\section{DOSAGE DE L'ACIDE LACTIQUE ET DES BASES DANS LE LAIT}

\section{Acide lactique}

Nous avons vu combien est simple dans cette méthode de VAN Slyke et PALMER, le dosage des acides organiques totaux libres ou salifiés dans une solution aqueuse. Il s'agit d'obtenir du lait une solution aqueuse débarrassée de tout ce qui pourrait gêner le dosage. Nous avons employé une défécation par l'hydrate de zinc (I) qui supprime les éléments gênants. Nous obtenons ainsi :

$1^{0}$ La précipitation totale des albumines, non seulement de la caséine mais encore de la lactalbumine. Cette méthode a déjà été 
employée pour déféquer les albumines du sérum sanguin en vue du dosage du sucre et de certains éléments minéraux.

20 Le zinc précipite également l'acide phosphorique très abondant dans le lait, - l'acide phosphorique étant un acide faible par sa forme phosphate mono basique serait dosé en partie par la méthode à l'Orangé IV.

$3^{\circ}$ Quand le lait fermenté a été neutralisé par des bicarbonates alcalins, la neutralisation de l'acide lactique ne va pas sans dégagement de $\mathrm{CO}^{2}$ qui, à titre faible, interviendrait également dans le dosage. Le $\mathrm{CO}^{2}$ est éliminé sous forme de carbonate de zinc insoluble

\section{Bases}

En réalité le dosage des acides organiques par la méthode de Van Slyke et Palmer est un dosage indirect, il ne mesure réellement que les bases liées aux acides organiques.

On peut donc envisager cette méthode au point de vue de la détermination des bases préexistant dans le lait. Si nous connaissons exactement la quantité d'alcalin que nous ajoutons au moment de la défécation pour amener le liquide à $p \mathbf{H} 8$ (rcse r htaléine), le dosage en retour par HCL mesurera cette quantité de bases ajoutées, plus les bases préexistant dans le lait; il suffira de soustraire l'alealin ajouté de l'alcalin mesuré pour avoir les bases préexistantes.

Nous indiquerons deux méthodes pour arriver à ce résultat, l'une combinée au dosage des acides organiques, l'autre pouvant mesurer les bases seules.

\section{Techniques}

\section{Dosage de l'acidité de titration.}

On prend $10 \mathrm{~cm}^{3}$ de lait bien homogène, que l'on met dans une éprouvette graduée. On ajoute de la phtaléine et de la soude N/10 en quantité suffísante pour virage au rose pâle.

Nous avons ainsi l'acidité de titration A.

\section{Dosage dês acides organiques.}

a) Défécation. On ajoute de l'eau distillée jusqu'à environ $20 \mathrm{~cm}^{3}$, puis on met $1 \mathrm{~cm}^{3} 5$ d'une solution concentrée commerciale de chlorure de zine $\mathrm{De}=1,45-45 \mathrm{Be}$, on agite ; on a par ailleurs déterminé la quantité de soude normale nécessaire pour amener au rose phtaléine $1 \mathrm{~cm}^{3} 5$ de la solution de chlorure de zinc, on ajoute cette quantité au mélange de lait et de $\mathrm{ZnCL}^{2}$, puis on amène le volume à $50 \mathrm{~cm}^{3}$ avec de l'eau distillée en agitant vigoureusement ; on laisse floculer, et on filtre. 
b) Dosages des acides organiques. On a préparé une solution d'Orangé IV dans l'eau distillée à $0,20 \%$. On dispose de deu x éprouvettes semblables jaugées à $60 \mathrm{~cm}^{3}$. Dans l'une on verse environ $25 \mathrm{~cm}^{3}$ d'eau distillée, $5 \mathrm{~cm}^{3}$ de la solution d'orangé IV et $1 \mathrm{em}^{3} 2$ d'HCl $\mathrm{N} / 10$, on complète à $60 \mathrm{~cm}^{3}$ avec de l'eau distillée. On obtient ainsi une teinte orangée qui servira de témoin.

Dans la seconde éprouvette, on verse $25 \mathrm{~cm}^{3}$ du filtrat après défécation, $5 \mathrm{~cm}^{3}$ d'orangé IV, on verse à la burette de l'HCl $\mathrm{N} / 10$ jusqu'à ce que la teinte de virage soit voisine de celle de l'étalon. On ajoute alternativement de l'eau et $\mathrm{HCl} \mathrm{N} / 10$ jusqu'à ce que cette teinte soit exactement celle du témoin avec un volume de $60 \mathrm{~cm}^{3}$.

On note le volume d' $\mathrm{HCl} \mathrm{N} / 10$ employé, on en soustrait $1 \mathrm{~cm}^{3} 2$ (nécessaire pour faire virer $60 \mathrm{~cm}^{3}$ d'eau à la teinte du témoin). Comme on a utilisé la valeur correspondante à $5 \mathrm{~cm}^{3}$ de lait, on multiplie par 200 pour obtenir la quantité d'acides organiques, contenue dans un litre de lait, exprimée en centimètre cubes de solution décinomale, on multiplie par 20 pour l'obtenir en valeur milimoléculaire.

\section{$3^{\circ}$ Dosage des bases libres liées aux acides organiques ou au $\mathrm{CO}^{2}$.}

Soit $\mathrm{A}=$ acidité ou titration pour $10 \mathrm{~cm}^{3}$ du liquide.

Soit $\mathrm{AO}=$ titration à l'orangé IV sur la moitié du filtrat.

Nous aurons : (AO $\left.-\frac{\mathrm{A}}{2}\right) 20$ millimols par litre.

On a done ainsi :

10 $\operatorname{Par} \mathbf{A}=$ acidité de titration; la quantité d'acides libres contenus dans le lait.

$2^{\circ}$ Par $\mathrm{AO}=$ la totalité des acides organiques.

$3^{\circ}$ Par $\mathrm{AO}-\mathrm{A}=$ la quantité des bases libres du lait.

Ce sont les bases surajoutées éventuellement au lait que nous dosons ainsi et non pas les bases constitutives des caséinates.

\section{Dosage des bases totales.}

Si on précipite la caséine par une dose faible, mais suffisante, d'acide acétique, et que l'on filtre le lactosérum, le dosage immédiat de ce lactosérum en présence d'orangé IV jusqu'à $p H$ 2,7 devrait être proportionnel aux bases.

Mais dans ces conditions la lactalbumine n'est pas retenue et R. GoIFFON et HAUDIQUET, ont montré que de très faibles quantités d'albumine empêcheraient le virage à l'Orangé IV de se faire à 
$p H \quad 2,7$, ils ont même indiqué que la quantité supplémentaire d' $\mathrm{HCl}$ au delà du $p \mathrm{H}$ réel 2,7 , pour-amener le colorant au virage correspondant à ce $p \mathrm{H}$, en solution aqueuse pure, pourrait être la somme des albumines complexes présentes. Cela rend ce procédé impossible à employer, parce qu'il mesurait à la fois les bases et la lactalbumine.

Il faut donc éliminer la lactalbumine par la chaleur après avoir précipité la caséine par l'acide acétique porté à l'ébullition avant de filtrer - le dosage devient alors possible, cependant l'expérience nous a prouvé que cette albumine ou bien n'était pas en quantité suffisante pour troubler le dosage, ou bien n'agissait pas sur le eolorant, c'est pourquoi ce temps de chauffage préalable, peut être éliminé.

\section{Dosage}

Il se fait de la façon suivante :

On prend $10 \mathrm{~cm}^{3}$ de lait bien homogène qu'on place dans une éprouvette graduée, on ajoute un peu 'd'eau distillée et de l'acide acétique pour précipiter la caséine, on amène le volume à $50 \mathrm{~cm}^{3}$, on place le précipité dans une fiole Erlenmeyer et on chauffe jusqu'à ébullition; on fait refroidir, on filtre $25 \mathrm{~cm}^{3}$ dans une éprouvette jaugée spécialement pour le dosage des acides organiques. On ajoute au filtrat $5 \mathrm{~cm}^{3}$ d'Orangé IV sans neutralisation préalable et on dose avec de l'HCl $\mathrm{N} / 10$, suivant la technique précédemment indiquée pour le dosage des acides organiques.

Nous obtenons ainsi les bases des caséinates débloquées par l'acide acétique et les bases éventuellement surajoutées.

\section{Résultats}

\begin{tabular}{|c|c|c|c|c|}
\hline- & $\begin{array}{c}\text { Acidité totale } \\
\text { par cent. cubes } \\
\text { N. } \%\end{array}$ & $\begin{array}{l}\text { Acides organi- } \\
\text { ques par cent. } \\
\text { cubes N. \%o }\end{array}$ & $\begin{array}{c}\text { Bases (par diffé- } \\
\text { rence) par cent. } \\
\text { cubes N. } \% \text { o }\end{array}$ & $\begin{array}{l}\text { Bases (dosage } \\
\text { direct) par cent. } \\
\text { cubes N. } \% \text {. }\end{array}$ \\
\hline Lait frais ....... & 18 & $\begin{array}{r}24 \\
-\quad 2\end{array}$ & 6 & 220 \\
\hline Lait frais . . . . . . . & 19 & 26 & 7 & 186 \\
\hline Lait frais . . . . . . & 17 & 22 & 4 & 192 \\
\hline Lait peu fermenté & 25 & 32 & 7 & 240 \\
\hline Lait peu fermenté & 46 & 47 & 1 & 173 \\
\hline Lait fermenté . . . & 85 & 87 & 2 & 210 \\
\hline Lait alcalinisé . . & 3 & 60 & $57-$ & 281 \\
\hline Lait alcalinisé et & & & & \\
\hline fermenté ..... & 40 & 110 & 70 & 276 \\
\hline Id. & 78 & 120 & 42 & 280 \\
\hline & & & & \\
\hline
\end{tabular}


Les bases libérées des protéinates l'emportent de beaucoup, comme ordre de grandeur, sur les bases liées aux acides organiques ; leur quantité est assez variable suivant les laits, soit que varie la quantité de caséine ou soit que la caséine contienne plus de bases.

Le procédé ne peut donc être employé pour le dépistage de l'alcalinisation frauduleuse du lait, ainsi qu'on le voit d'après le tableau ci-dessus. On peut tout au plus envisager que, joint au dosage de la matière grasse et du lactose, l'abaissement des chiffres indiqué par ce tableau pourrait donner un indice supplémentaire de mouillage.

\title{
DIFFICULTÉS RENCONTRÉES DANS LE DIAGNOSTIC DE LA MAMMITE STREPTOCOCCIQUE CONTAGIEUSE DES VACHES LAITIERES (1)
}

\author{
par
}

\section{COTONI, P. FORGEOT et G. THIEULIN}

Des recherches anciennes sur les divers streptocoques nous ont conduits à étudier depuis plusieurs années la mammite streptococcique des vaches laitières. L'importance économique de cette affection de nature chronique est connue dans le monde entier. Les chiffres suivants suffisent à donner une idée des pertes que cause la maladie. STABLEFORTH (2) a estimé la perte annuelle en lait de vache subie, du fait de la mammite contagieuse, en Angleterre et au Pays de Galles réunis, à 3.240.000 livres sterling. Le Dr Stwiger (3), Ministre de l'Agriculture, parlant en 1941, devant une commission du Landtag prussien, au cours de la discussion du budget de l'Agriculture, évaluait, d'après une estimation prudente, les dommages imputables à la mam mite streptococcique, à 250.000 .000 de marks-or par an.

La lecture de nombreux travaux sur le traitement, impose la notion de l'efficacité restreinte des diverses méthodes thérapeutiques proposées. Ne se heurte-t-on pas souvent au caractère invétéré des lésions? La solution du problème ne résiderait-elle pas dans l'établissement d'un diagnostic très précoce, à une époque où les signes cliniques, obscurs, incertains, absents même, ne permettent pas de reconnaître la mammite? Il n'est pas défendu d'espérer qu'on pourrait, à cette époque-là, agir efficacement, grâce à la chimiothérapie, et arrêter l'éclosion d'épidémies par un sévère isolement

(1) Soc. fr. Microbiologie, 4 novembre 1948. In Annales Institut Pasteur, 1949, $76,76$.

(2) Stableforth. Proceed. Roy. Soc, Med., 1942, 35, 625.

(3) Steiger, cité par Poreher. Le Lait, 1932, 12, n० 114, 257. 